\title{
Quick extraction and direct determination of amino acids from plants by hydrophilic interaction liquid chromatography (HILIC) and high-performance liquid chromatography-mass spectrometry without derivatization
}

\author{
Xuemei Chen ${ }^{1}$, Dejian Feng ${ }^{2}$, Yan Zou ${ }^{2}$, Huaiping $\mathrm{Li}^{2^{*}}$, and Hang Song ${ }^{1 *}$ \\ ${ }^{1}$ School of Chemical Engineering, Sichuan University, Chengdu 610065, China \\ ${ }^{2}$ National Institute of Measurement and Testing Technology, Chengdu 610021, China
}

\begin{abstract}
Amino acids are naturally compound in many plants and have a essential effects on human life. This work presents a simple and rapid analytical method for direct determination of amino acids without derivatization. The preparation method of samples was green and quick, which extracted amino acids with water in 10 min, and separated on a HILIC-Z (2.1 $\mathrm{mm} \times 100 \mathrm{~mm}, 2.7 \mu \mathrm{m}$ i.d.) using acetonitrile-water solution as mobile phase, and then detected under ESImultiple reaction monitoring mode. Satisfactory determination of 21 underivatized amino acids from three different plants was achieved in $15 \mathrm{~min}$. This method showed a good linearity $\left(\mathrm{R}^{2}>0.99\right)$ for the analyst and the detection limits (LOD) were $0.53 \mathrm{mg} / \mathrm{kg}-10.39 \mathrm{mg} / \mathrm{kg}$ for the amino acids. The average recoveries were in the range of $80 \%-120 \%$ at spiked concentrations. The analytical method is high sensitivity, accuracy and analytical efficiency, meanwhile it may be used for the analysis of amino acids in other plants.
\end{abstract}

\section{Introduction}

Amino acids (AAs) are important nutrients and ingredients for life, meanwhile, AAs are important compounds[1] to maintain vital body functions including protein synthesis, healthy growth, tissue repair and balanced metabolism $[2,3]$. The most of AAs, except tryptophan, phenylalanine and tyrosine, lack a strong chromophore [4], such as no ultraviolet absorption and no fluorescence[3]. Therefore, AAs are always determined by derivatization to increase detection sensitivity, but there are some drawbacks, such as complex derivatization procedure, incomplete derivatization, derivative instability, reagent interference $[5,6]$, expensive reagents $[7,8]$ and long analysis time $[9,10]$. Therefore, a quick, simple and accurate analytical method for amino acid is needed.

This study explored a green, quick extraction method and developed an efficient, sensitive determination method by using HILIC-HPLC-ESI-MS/MS for simultaneous quantification of free amino acids (FAAs) in three plants. The extraction proceeded with water in short time and the sample extraction conditions were optimized using orthogonal experiment. Moreover, important analytical conditions such as gradient elution procedures, eluent composition including $\mathrm{pH}$ and salt additive concentration were investigated.

\section{Experimental}

\subsection{Material and Instruments}

All 21 amino acid standard products were purchased from Sigma-Aldrich (Shanghai, China) and their purities are higher than $98 \%$. Acetonitrile, formic acid, ammonium formate and concentrated hydrochloric acid were purchased from Chengdu Kelon Chemical Reagent Co., Ltd. The water was prepared using a Milli-Q purification system (Millipore). The water bath box (DK-8AX) was purchased from Shanghai Precision Instrument Co., Ltd (Shanghai, China). The high-speed grinder was purchased from China Yongkang Shengxiang Electric Co., Ltd (Zhejiang, China).

\subsection{Standard solutions and sample preparation}

Stock solutions of each amino acid standard were prepared at a concentration of $100 \mathrm{mg} / \mathrm{mL}$ in $0.1 \mathrm{~N}$ hydrochloric acid and stored at $4^{\circ} \mathrm{C}$. Working standards were prepared from the stock solutions by appropriate dilution with acetonitrile/water 50:50 (v/v). Ammonium formate solution ( $\mathrm{pH} \mathrm{3.0;100} \mathrm{mM),} \mathrm{used} \mathrm{as} \mathrm{a} \mathrm{component} \mathrm{of} \mathrm{the}$ mobile phase in HILIC separations, was prepared by dissolving reagent in water and adding formic acid up to the $\mathrm{pH}$ 3.0. Solutions were passed through a $0.45 \mu \mathrm{m}$ membrane filter and stored at $4^{\circ} \mathrm{C}$.

*Corresponding author: Huaiping Li: likaicyo@163.com; Hang Song: hangsong@ vip.sina.com 
Tea samples were purchased from a local market in Chengdu, Sichuan Province, China. The samples were pulverized to homogeneous powders (40 mesh) and $0.5 \mathrm{~g}$ was added into $250 \mathrm{~mL}$ conical flask. Then $100 \mathrm{~mL}$ of hot water $\left(80^{\circ} \mathrm{C}\right)$ was added and extracted with water bath for $10 \mathrm{~min}$ at $80^{\circ} \mathrm{C}$. The solid-liquid mixture was filtrated after extraction. The filtrate was cooled to room temperature and centrifuged at $14000 \mathrm{r} / \mathrm{min}$ for $10 \mathrm{~min}$ at $4^{\circ} \mathrm{C}$ to obtain supernatants for injection.

\subsection{Analytical condition}

HPLC-MS/MS system includes a 6465-mass spectrometer Apparatus and an Agilent 1290 UHPLC system (Agilent Technologies, SantaClara, CA, USA). Agilent HILIC-Z column $(2.1 \mathrm{~mm} \times 100 \mathrm{~mm}, 2.7 \mu \mathrm{m}$ i.d. $)$ was used as the stationary phase. HPLC system was connected to a mass spectrometer equipped with an ESI interface operating in the positive ion mode. Chromatography separation was carried on at $25{ }^{\circ} \mathrm{C}$. The mobile phase $\mathrm{A}$ and $\mathrm{B}$ are $10 \%$ $200 \mathrm{mmol} / \mathrm{L}$ ammonium formate-water solution $(\mathrm{pH}=3.0)$ and $10 \% 200 \mathrm{mmol} / \mathrm{L}$ ammonium formate-acetonitrile solution $(\mathrm{pH}=3.0)$, respectively. Different gradient elution was investigated and then finally optimized with following program:

0 min-11.5 $\min , 100 \%-70 \% \mathrm{~B} ; 11.5 \mathrm{~min}-12 \mathrm{~min}$, $70 \%-100 \% \mathrm{~B} ; 12 \mathrm{~min}-25 \mathrm{~min}, 100 \%-100 \% \mathrm{~B}$. The flow rate was $0.5 \mathrm{~mL} / \mathrm{min}$, and the sample injection volume was $1 \mu \mathrm{L}$. The operating parameters included as follows: drying gas temperature, $230^{\circ} \mathrm{C}$; nebulizer gas (N2), $20 \mathrm{psi}$; drying gas (N2) flow, $11 \mathrm{~L} / \mathrm{min}$; sheath temperature, $390^{\circ} \mathrm{C}$; sheath flow, $12 \mathrm{~L} / \mathrm{min}$; spray pressure, $1500 \mathrm{~V}$. MS chromatograms were obtained with a mass range of 0 and $300 \mathrm{mz}$ and a scan rate of $615 \mathrm{~ms} /$ cycle.

\section{Results and discussion}

\subsection{Optimization of the sample preparation}

The conditions of sample preparation were optimized by orthogonal experiment and the results are shown in Table 1 . The best condition is temperature $80^{\circ} \mathrm{C}$, extraction time $10 \mathrm{~min}$, material liquid ratio 0.5:100, particle size 40, respectively. Particle size is the most important parameter, and 40 mesh is better than others. More tiny particles were easier to float in the surface layer of extraction solvent and thus could limit completed contact between particles and solvent [11].

Table 1 Orthogonal design and experimental results of sample

\begin{tabular}{llllll}
\hline \multicolumn{7}{c}{ extraction } \\
No & $\begin{array}{l}\text { Temp } \\
\left({ }^{\circ} \mathrm{C}\right)\end{array}$ & $\begin{array}{l}\text { Time } \\
(\mathrm{min})\end{array}$ & $\begin{array}{l}\text { Material } \\
\text {-liquid } \\
\text { ratio } \\
(\mathrm{n}: 100)\end{array}$ & $\begin{array}{l}\text { Particle } \\
\text { size } \\
(\text { mesh })\end{array}$ & $\begin{array}{l}\text { total } \\
\text { amino } \\
\text { acids } \\
(\%)\end{array}$ \\
\hline 1 & 60 & 5 & 0.5 & 40 & 1.49 \\
2 & 60 & 10 & 1 & 60 & 1.30 \\
3 & 60 & 20 & 2 & 80 & 1.16 \\
4 & 80 & 5 & 1 & 80 & 1.24 \\
5 & 80 & 10 & 2 & 40 & 1.51 \\
6 & 80 & 20 & 0.5 & 60 & 1.37 \\
7 & 100 & 5 & 2 & 60 & 1.23 \\
\hline
\end{tabular}

\begin{tabular}{llllll}
\hline 8 & 100 & 10 & 0.5 & 80 & 1.30 \\
9 & 100 & 20 & 1 & 40 & 1.58 \\
$\mathrm{~K} 1$ & 1.31 & 1.32 & 1.39 & 1.53 & \\
$\mathrm{~K} 2$ & 1.37 & 1,37 & 1.37 & 1.30 & \\
$\mathrm{~K} 3$ & 1.37 & 1.37 & 1.30 & 1.23 & \\
$\mathrm{R}$ & 0.06 & 0.08 & 0.09 & 0.29 & \\
\hline
\end{tabular}

\subsection{Operation conditions of liquid chromatography}

Different gradient elution procedures will have an important impact on the separation effect, so different mobile phase elution gradients are studied. The elution method is as follows: (1) The mobile phase B is eluted from $100 \%$ to $70 \%$ within 8 minutes, and all amino acids were separated within 9 minutes. (2) The mobile phase B is eluted from $100 \%$ to $70 \%$ within 11.5 minutes, and all amino acids were separated within 11 minutes; (3) The mobile phase B is eluted from $100 \%$ to $70 \%$ within 15 minutes, and all amino acids are separated within 13 minutes; (4) The mobile phase B is eluted from $100 \%$ to $70 \%$ within 20 minutes, and all amino acids are separated within 15 minutes. The peak time of each amino acid increased with the change time of the mobile phase, but there was no significant difference in response. The resolution of $\gamma$-aminobutyric acid and theanine increased with the decrease of the organic proportion, while the resolution of other amino acids did not change much. In order to improve analysis efficiency and achieve better separation of amino acids, the mobile phase B is eluted from $100 \%$ to $70 \%$ within 20 minutes. According to the research of Wu Ze ying[12], in HILIC, the lower the water content, the greater the retention of the analyte. Therefore, the separation effect of the flow gradient (4) is better than others.

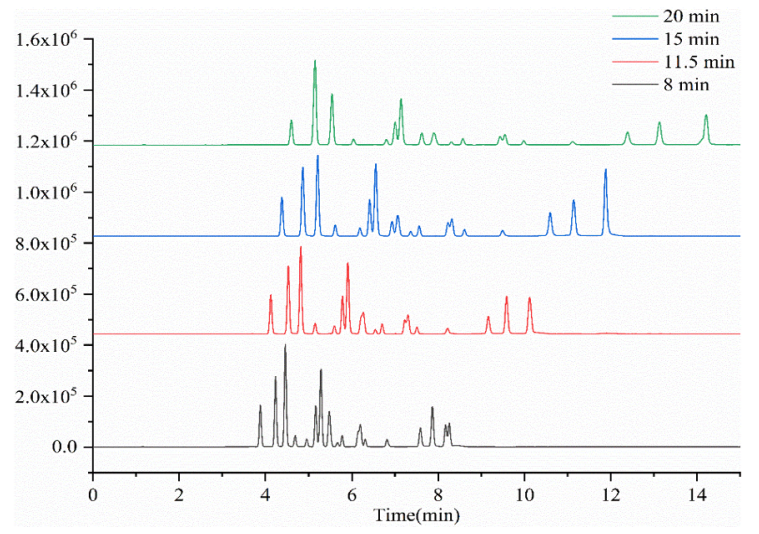

Figure 1 Comparison of 3 chromatographic columns

Ammonium formate as salt additive added in the mobile phase may improve peak shapes for amino acids compared to other additives [2]. Ammonium formate of mobile phases $A$ and $B$ can maintain the acidic environment of separation system that can improve the separation effect and sensitivity. It was reported [13, 14] that the concentration of salt additive also influences the retention. Therefore, the ammonium formate concentration of $50 \mathrm{mmol} / \mathrm{L}-300 \mathrm{mmol} / \mathrm{L}$ was investigated and the result is shown in Figure 2. When the concentration of ammonium formate increased from 50 $\mathrm{mmol} / \mathrm{L}$ to $100 \mathrm{mmol} / \mathrm{L}$, the separation effect of tyrosine, valine and proline decreased, while the separation effect 
of threonine, glycine, glutamine, serine and asparagine increased, and the response effect of histidine, cystine, lysine and arginine increased; for the concentration range from $100 \mathrm{mmol} / \mathrm{L}$ - $200 \mathrm{mmol} / \mathrm{L}$, Alanine and threonine could not be separated, and the response effect of histidine, cystine, lysine and arginine reduced; the separation effect of amino acids gradually decreased after $200 \mathrm{mmol} / \mathrm{L}$. So, the $100 \mathrm{mmol} / \mathrm{L}$ was selected.

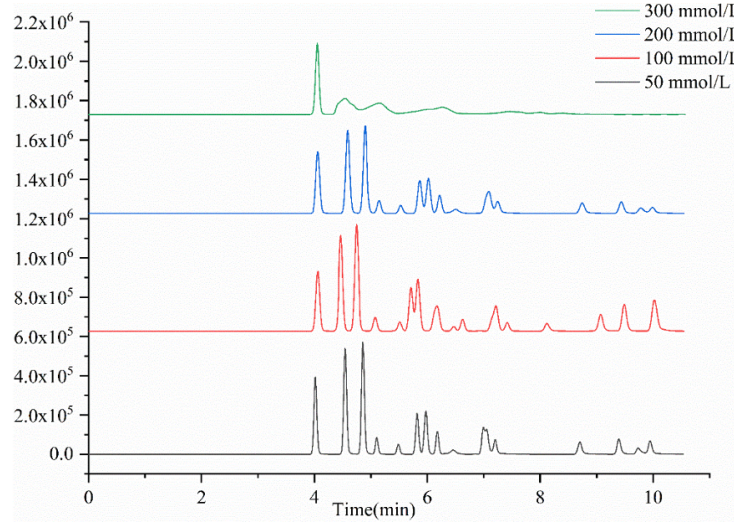

Figure 2 Comparison of 4 ammonium formate concentration chromatograms

The retention and selectivity can be influenced by $\mathrm{pH}$ [14]. Hence the $\mathrm{pH}$ effect was investigated, and the result is shown in Figure 3. The response of each amino acid had not significantly change in $\mathrm{pH} 2.0$ to $\mathrm{pH} 3.0$, while the separation effect of each amino acid increased. However, the separation and response effect of each amino acid had not significantly changed in $\mathrm{pH} 3.0$ to $\mathrm{pH} 3.5$. It is the same as the study of Ze-Ying Wu (2013) [12]. In our study, the $\mathrm{pH} 3.0$ was selected.

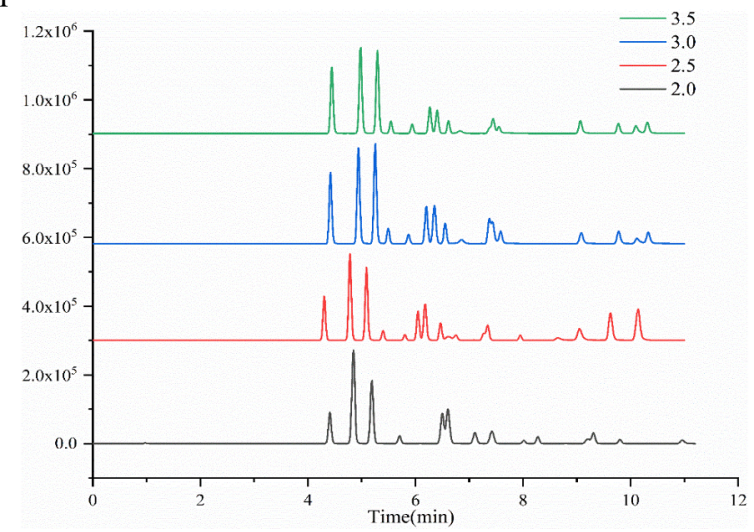

Figure 3 The comparison of $4 \mathrm{pH}$ chromatogram

Other liquid chromatography conditions such as flow rate, injection volume and column temperature were optimized. The final conditions were the flow rate, injection volume and column temperature were determined to be $0.5 \mathrm{~mL} / \mathrm{min}, 1 \mu \mathrm{L}$ and $25^{\circ} \mathrm{C}$, respectively.

MS/MS parameters were optimized by direct injection $1 \mu \mathrm{g} / \mathrm{mL}$ standard solution of each amino acid at a flow rate of $1 \mathrm{~mL} / \mathrm{min}$. Solutions were prepared and injected into the ESI source in positive and negative modes in the range of $0-300 \mathrm{~m} / \mathrm{z}$. All amino acids provided the best response in ESI positive ion mode, so their quasimolecular ions $[\mathrm{M}+\mathrm{H}]^{+}$could be observed. The optimal selective reaction monitoring (SRM) transitions for each amino acid was investigated using fragmentor (from 40 to $100 \mathrm{~V}$ ) and collision energies (from 0 to $50 \mathrm{~V}$ ). The mass parameters that are used to choose the mass detection and quantitate the most intense ions (base ion) are shown in Table 2. Based on the chromatographic and ESI-MS/MS conditions described above, a satisfactory separation can be obtained in about 15 min (Figure 4).

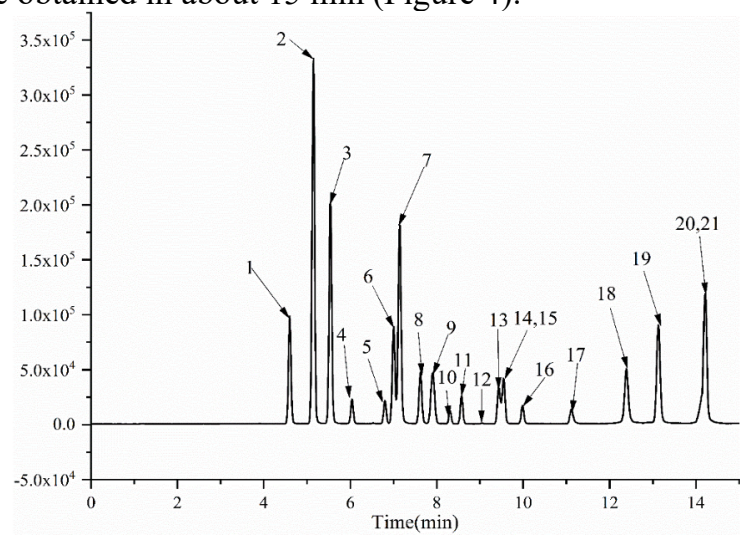

Figure 421 amino acids chromatograms (1: Phenylalanine (Phe); 2: Isoleucine (Iso); 3: Leucine (Leu); 4: Methionine (Met); 5: Tyrosine (Tyr); 6: Valine (Val); 7: Proline (Pro); 8: $\gamma$ aminobutyric acid (GABA); 9: Theanine (The); 10: Alanine (Ala); 11: Threonine (Thr); 12: Glycine (Gly); 13: Glutamine (Glu); 14: Serine (Ser); 15: Asparagine (Asp); 16: Glutamic (Glu); 17: Aspartic (Asp); 18: Histidine (His); 19: Arginine (Arg); 20: Cystine (Cys); 21: Lysine (Lys)) 
Table 2 The Mass spectrometry parameters, linear range, $\mathrm{R}^{2}$, LOD, average recoveries, precision and stability of 21 amino acids

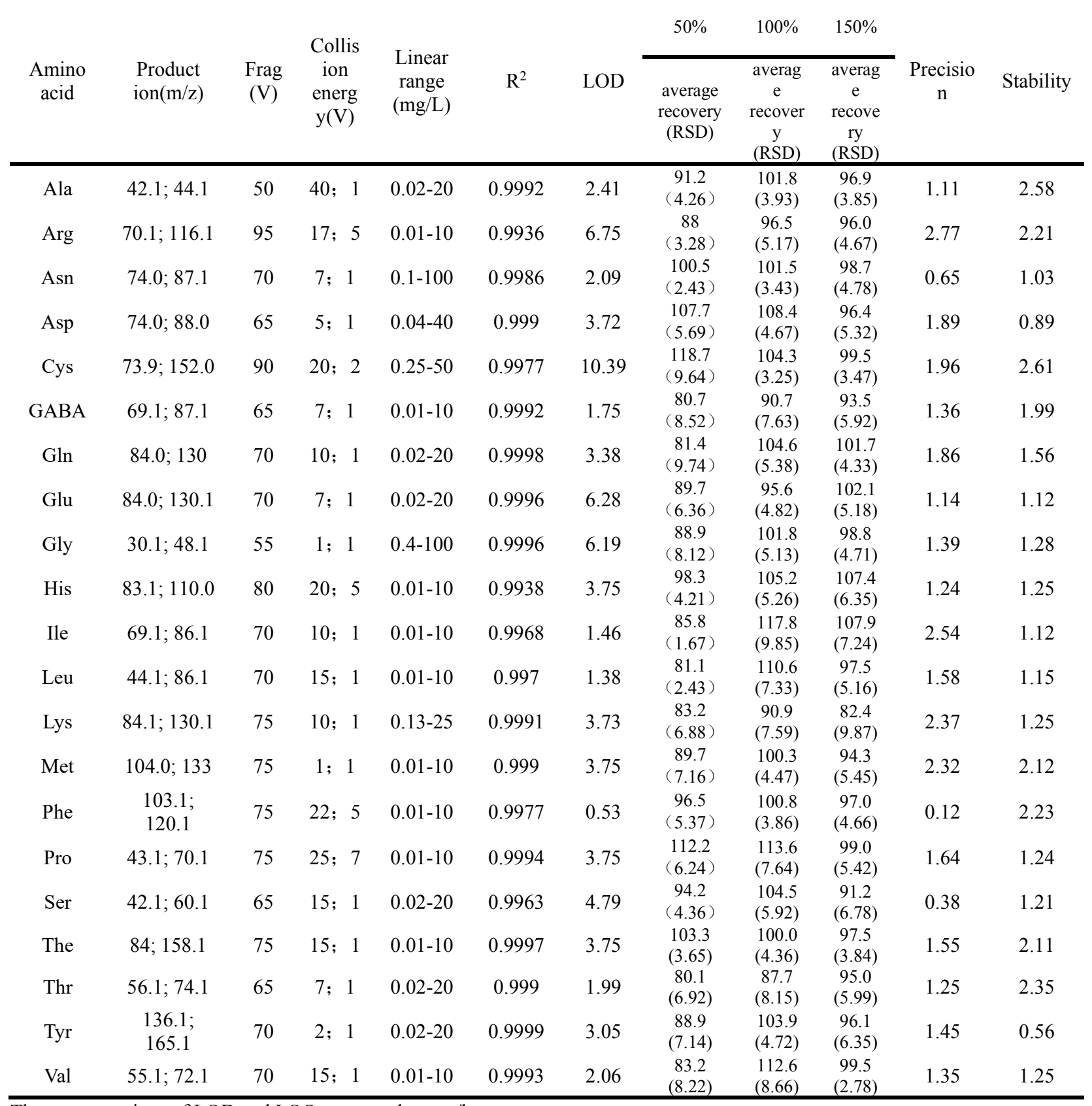

The concentrations of LOD and LOQ expressed as $\mathrm{mg} / \mathrm{kg}$.

\subsection{Linearity range, $R^{2}$, LOD}

Under the chromatographic conditions described above, the calibration graphs were constructed with the ratio of each amino acid peak area and concentration. Good linearity was achieved in the range studied for each organic acid and determination coefficients $\left(\mathrm{R}^{2}\right)$ is higher than 0.99 .

Since the presence of matrix effect and the fact that no blank matrices are available in most cases, LOD was estimated by extrapolation of the $\mathrm{S} / \mathrm{N}$ of the extract with known amounts of analytes [15]. The LOD is determined as the amount for which the signal-to-noise ratio $(\mathrm{S} / \mathrm{N})$ was 3 . The results of LOD for the 21 amino acids are listed in Table 2. The LOD was in the ranges of $0.53 \mathrm{mg} / \mathrm{kg}-$ $10.39 \mathrm{mg} / \mathrm{kg}$.

\subsection{Recovery, precision and stability}

The accuracy was measured by recovery experiments performed on each of the analytes that were spiked to the real samples at three levels $(50 \%, 100 \%$ and $150 \%)$. The $100 \%$ spiked amount of aspartic acid, glutamine and glutamate is $1000 \mathrm{mg} / \mathrm{kg}$; the $100 \%$ spiked amount of theanine is $15000 \mathrm{mg} / \mathrm{kg}$; the $100 \%$ spiked amount of other amino acids is $400 \mathrm{mg} / \mathrm{kg}$. Table 1 shows the average recovery are in the range of $80 \%-120 \%$ and the RSDs are lower than $10 \%$ (Table 2). The results indicated that the method has good accuracy in the determination of 21 AAs of this method.

Six tea samples were accurate weighed, prepared and analyzed by HPLC-MS/MS. The precision results are shown in Table 2. The RSD of free AAs in tea samples 
were all less than $2.77 \%$, indicating that this method has good precision.

A tea sample was accurate weighed and prepared, and the sample was analyzed at $0,2,4,8,16$ and 24 hours, respectively. The stability results are shown in Table 2 . The RSD of AAs in tea samples were all less than $2.58 \%$, indicating that this method has good stability.

\section{Conclusions}

A direct method for determination of amino acids in plants was established with HILIC and HPLC-MS/MS for amino acid determination. The sample extraction with water could be completed in $10 \mathrm{~min}$, hence it is simpler, faster and more environmentally friendly. Determination process of 21 underivatized AAs from plants was completed in 15 min with satisfactory recovery, precision and stability. Therefore, this method is reliable and effective that can quantitative analysis 21 AAs without derivatization in plants.

\section{Acknowlegements}

This work was supported by National Standard Project (20171129-T-469).

\section{References}

1. Yao, X., et al. J Sep Sci 36, 17(2013)

2. Guo, S., et al. J Agric Food Chem 61, 11(2013)

3. Kazan, R.M., et al. Microchem J 150(2019)

4. Kato, M., et al., Accredit Qual Assur 18, 6(2013)

5. Thiele, B., et al. Methods Mol Biol 828(2012)

6. Liyanaarachchi, G.V.V., et al. J Chromatogr A 1568(2018)

7. Warren, C. Soil Biol Biochem 40, 4(2008)

8. Mustafa, A., et al. Food Chem 105, 1(2007)

9. Luo, T., et al. Electrophoresis 39, 11(2018)

10. Omar, M.M.A., A.A. Elbashir, and O.J. Schmitz. Food Chem 214(2017)

11. Abdulbari, H.A., et al. MATEC Web of Conferences 111(2017)

12. Wu, Z.-Y., et al. J SEP SCI 36, 14(2013)

13. Kalíková, K., et al. Chem Pap 72, 1(2017)

14. Guo, Y. and S. Gaiki. J Chromatogr A 1074, 1-2(2005)

15. Flores, P., P. Hellín, and J. Fenoll. Food Chem 132, 2(2012) 